\title{
A model for ranking and selecting integrity tests in a distributed database
}

\begin{abstract}
Checking the consistency of a database state generally involves the execution of integrity tests on the database, which verify whether the database is satisfying its constraints or not. This paper presents the various types of integrity tests as reported in previous works and discusses how these tests can significantly improve the performance of the constraint checking mechanisms without limiting to a certain type of test. Having these test alternatives and selecting the most suitable test is an issue that needs to be tackled. In this regard, the authors propose a model to rank and select the suitable test to be evaluated given several alternative tests. The model uses the amount of data transferred across the network, the number of sites involved, and the amount of data accessed as the parameters in deciding the suitable test. Several analyses have been performed to evaluate the proposed model, and results show that the model achieves a higher percentage of local processing as compared to the previous selected strategies.
\end{abstract}

Keyword: Distributed Database, Integrity Constraints, Integrity Constraints Checking, Integrity Tests, Performance 